\title{
PERDA TÉRMICA NA PANELA DE FERRO-GUSA THYSSENKRUPP CSA*
}

José Artur Albano de Guimarães ${ }^{1}$ Daniel Augusto Godinho de Carvalho Marcos Viana ${ }^{3}$ Leonardo Demuner ${ }^{4}$ Caio Nogueira Araújo Diniz ${ }^{5}$

\begin{abstract}
Resumo
A temperatura de carregamento do ferro-gusa no convertedor é de suma importância para 0 acerto do balanço térmico do convertedor. Dessa maneira, o presente trabalho teve como objetivo a proposição de uma equação de modelamento da perda térmica na panela de ferro-gusa da thyssenkrupp CSA com o intuito de prever, com melhor acurácia, a temperatura de carregamento no convertedor. Os resultados encontrados pelo modelo foram comparados com os valores reais de perda térmica na panela e concluiu-se que a equação desenvolvida se mostrou bastante assertiva e abrangente. Foram consideradas novas variáveis nesta abordagem que, até então, não eram utilizadas pelo modelo de perda térmica nas panelas de ferro-gusa da CSA.
\end{abstract}

Palavras-chave:Panela; Ferro-gusa; Perda térmica; Convertedor

\section{THERMAL LOSS MODEL IN THYSSENKRUPP CSA PIG IRON LADDLE}

\begin{abstract}
The charging temperature of hot metal at BOF converter is one of the key factors for the BOF process thermal balance adjustment. In this way, the present work had the objective of proposing a thermal loss equation in the hot metal ladle of thyssenkrupp CSA in order to predict the charge temperature in the converter. A comparison between the current and proposed model were conducted. The new approach showed a comprehensive and wider thermal losses description for the hot metal ladle. New variables were implemented in this new approach which, until then, were not used by the thermal loss model in thyssenkrupp CSA hot metal laddle.i

Keywords: Laddle, Hot metal; Thermal Loss; Conversor

1 Estudante de Engenharia Metalúrgica, Universidade Federal de Minas Gerais, Belo Horizonte, Minas Gerais, Brasil.

2 Engenheiro Aciaria, thyssenkrupp CSA, Rio de Janeiro, Rio de Janeiro, Brasil.

3 Engenheiro Aciaria, thyssenkrupp CSA, Rio de Janeiro, Rio de Janeiro, Brasil.

4 Gerente Geral Aciaria, thyssenkrupp CSA, Rio de Janeiro, Rio de Janeiro, Brasil.

5 Engenheiro de qualidade de placas, thyssenkrupp CSA, Rio de Janeiro, Rio de Janeiro, Brasil.
\end{abstract}




\section{INTRODUÇÃO}

Em meio a um mercado siderúrgico bastante competitivo e desafiador, as usinas nacionais e internacionais têm buscado novas tecnologias e alternativas para 0 processo. Dentre outros, a razão pela qual as empresas buscam melhorias é a demanda dos clientes por aços de maior qualidade a um preço razoável. Porém, para se produzir um aço de alta qualidade, faz-se necessário um rigoroso conhecimento e, consequentemente, controle do processo.

O presente trabalho foi realizado na área da aciaria da thyssenkrupp CSA, localizada na cidade do Rio de Janeiro. A aciaria da CSA, na área do refino primário, consiste em duas estações de dessulfuração do ferro-gusa e em dois conversores de 330 toneladas de capacidade. Dentre outros, um problema recorrente na usina é a previsão da temperatura de carregamento do ferro-gusa no conversor LD. Devido à ausência de um sistema de medição de temperatura pré-carregamento, faz-se uma previsão da temperatura do ferro-gusa por meio de uma taxa linear de perda térmica fixa. Portanto, a temperatura prevista para o ferro-gusa é obtida pela diferença da temperatura medida no final do processo de dessulfuração e da perda térmica calculada a partir do tempo em que a panela de ferro-gusa esperou até ser carregada no conversor.

O cálculo da temperatura do ferro-gusa pré-carregamento é de suma importância para a estimativa correta do balanço térmico do conversor realizada pelo modelo estático dos conversores. Por meio do balanço térmico, é calculada, entre outras, a quantidade de materiais refrigerantes a ser adicionada no conversor. Portanto, um erro na temperatura inicial do ferro-gusa leva a um erro no cálculo destes refrigerantes, por exemplo. A adição de menores ou maiores quantidades de refrigerante afeta a temperatura final de sopro, acarretando, assim, vários problemas na corrida.

Dessa maneira, como objetivo do presente trabalho, destaca-se a análise do modelo de perda térmica na panela de gusa (taxa) utilizado atualmente na aciaria da thyssenkrupp CSA e a proposta de um modelo mais abrangente e assertivo com o intuito de aumentar a precisão do cálculo de sopro do BOF. De certa maneira, a proposta considerou a adição de novos parâmetros relacionados à perda térmica na panela de gusa como, por exemplo, o peso bruto da panela, a temperatura do ferrogusa imediatamente após a dessulfuração, o tempo em que a panela permaneceu sem gusa, o tempo de espera da panela para o carregamento e a vida da panela. A figura 1 ilustra a influência da vida da panela na perda térmica em uma panela de aço, situação bastante similar à ocorrente em uma panela de gusa, segundo Ferreira et al (2004) [1]. Utilizando simulação numérica de escoamento e transferência de calor (CFD), os autores avaliaram a influência do desgaste de panelas sobre as perdas térmicas do aço líquido. Simulando uma panela em final de campanha com $80 \mathrm{~mm}$ de desgaste em relação à nova, conclui-se que as perdas térmicas do aço líquido para os refratários são maiores na panela de vida elevada nos primeiros 15 minutos de permanência Após este período a situação se reverte, e a perda é maior na panela nova. Este comportamento se deve ao maior potencial de perda pela parede lateral apresentado pela panela velha. Este potencial de perda é função do histórico térmico da panela. 


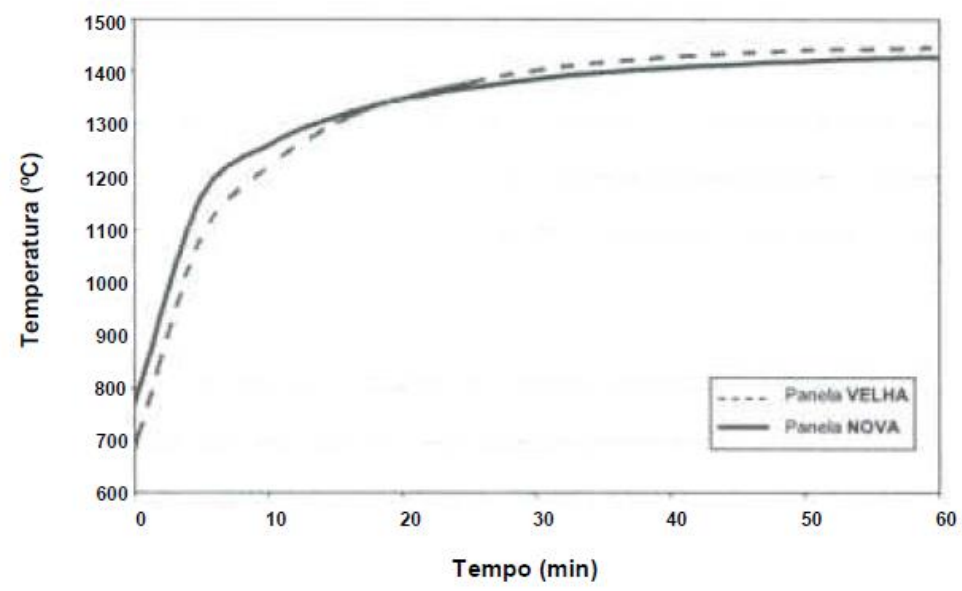

Figura 1: Temperatura média na camada de $50 \mathrm{~mm}$ mais próxima à área de contato do aço, segundo Ferreira et al (2004)

A perda térmica na panela de ferro-gusa está relacionada a diversos aspectos. Dentre eles, pode-se destacar: calor conduzido através da camada de escória e transferido para o ambiente por radiação e convecção, espessura da escória e transferência de calor para o revestimento refratário [2]. Através de um modelo matemático unidimensional, Mundimet al (1985) [3] avaliaram a influência da espessura da parede refratária sobre as perdas térmicas. Os resultados são mostrados na figura 2. Nota-se que à medida que a espessura da parede refratária diminui, as perdas térmicas e a queda de temperatura do aço aumentam.

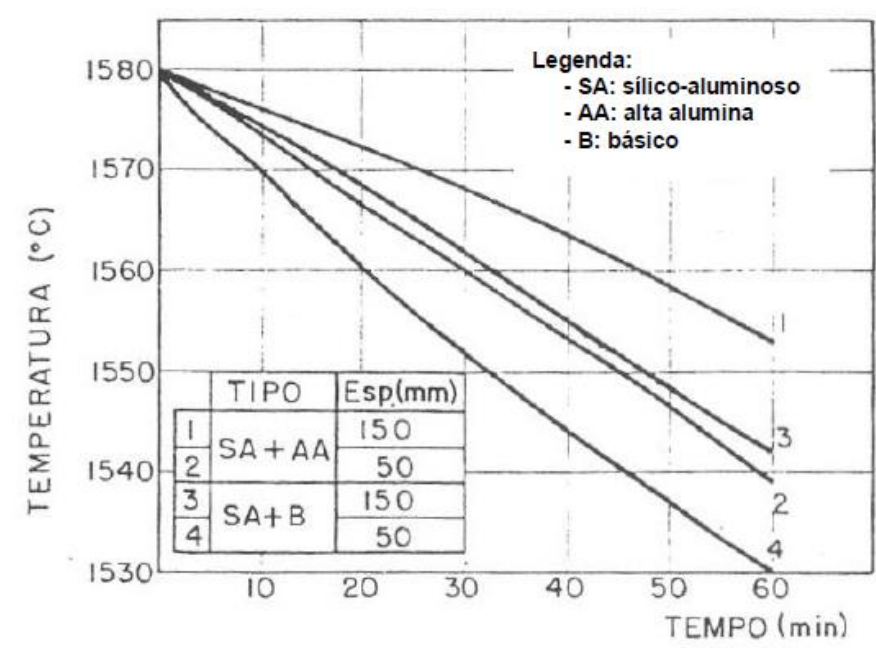

Figura 2: Efeito do tipo e espessura do revestimento refratário sobre a evolução da temperatura do aço em uma panela de $180 \mathrm{t}$, segundo Mundimet al (1986)

A figura 3 ressalta a influência da espessura da camada de escória na perda térmica. Pode-se observar que a temperatura do líquido diminui mais acentuadamente no caso de uma escória de camada fina [4]. Ao contrário, com o aumento da espessura da escória, há a diminuição da perda térmica. 


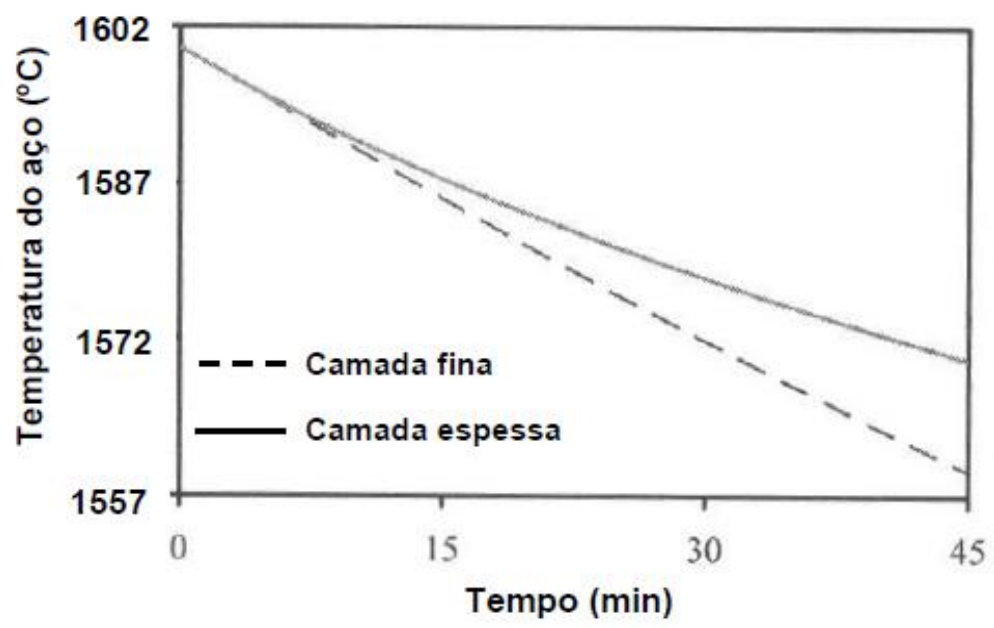

Figura 3: Variação da temperatura do aço líquido ao longo do tempo para duas condições de espessura de escória, segundo Tetraultet al (2004)

A figura 4 mostra as principais formas de perda térmica na panela de aço, que é similar ao que ocorre na panela de gusa.

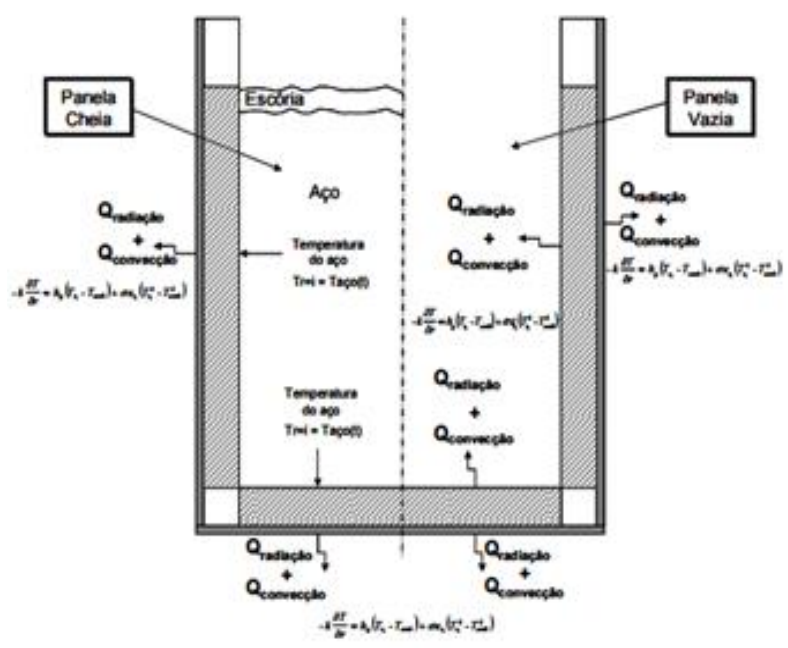

Figura 4: Principais formas de perda térmica na panela de aço (

Porém, devido à complexidade das equações diferenciais que regem a radiação, convecção e condução, optou-se pela proposta, no presente trabalho, de um modelo estatístico de perda térmica na panela de gusa da thyssenkrupp CSA.

\section{MATERIAIS E MÉTODOS}

O presente trabalho foi realizado na aciaria da thyssenkrupp CSA. Com o intuito de propor uma equação estatística de perda térmica considerando os parâmetros vida da panela (número de corridas), tempo de espera para carregamento da panela (minutos), tempo da panela sem gusa (minutos), peso bruto da panela (toneladas) e temperatura do gusa imediatamente após a dessulfuração $\left({ }^{\circ} \mathrm{C}\right)$, foi necessário uma ampla coleta de dados na área. Após o término do tratamento de dessulfuração, a panela é liberada da estação e a ponte rolante a transporta para um cavalete onde a 
mesma espera até o carregamento. Para a realização deste trabalho, foi necessário que a ponte rolante transportasse, em intervalos de trinta minutos, a panela até a estação de dessulfuração para medir novamente a temperatura do ferro-gusa. A partir destas medições, pôde-se calcular a perda térmica do ferro-gusa em um intervalo de tempo conhecido. Durante dois meses de coleta de dados, foi possível obter dados suficientes para a realização de um tratamento estatístico.

Além disso, foi possível obter, no sistema online da CSA, os outros parâmetros necessários para a montagem do modelo de perda térmica proposto. Foi construída, então, a tabela $1 \mathrm{com}$ os dados necessários para realizar o tratamento estatístico, em que $\Delta T$ é a perda térmica, Vida é a vida da panela, Peso bruto é o peso bruto da panela de gusa, Tempo sem gusa é o tempo da panela sem gusa, Temperatura inicial é a temperatura do gusa imediatamente após a dessulfuração e tempo de espera é o tempo de espera da panela para carregamento. Foram coletadas 48 observações.

Tabela 1: Dados coletados para modelamento da equação de perda térmica

\begin{tabular}{|c|c|c|c|c|c|}
\hline$\Delta \mathrm{T}\left({ }^{\circ} \mathrm{C}\right)$ & Vida & $\begin{array}{l}\text { Peso bruto } \\
\text { (t) }\end{array}$ & $\begin{array}{l}\text { Tempo sem gusa } \\
\text { (min) }\end{array}$ & $\begin{array}{c}\text { Temperatura inicial } \\
\left({ }^{\circ} \mathrm{C}\right)\end{array}$ & $\begin{array}{l}\text { tempo de espera } \\
\text { (min) }\end{array}$ \\
\hline$\Delta \mathrm{T}_{1}$ & $V_{1}$ & $\mathrm{P}_{1}$ & $\mathrm{Ts}_{1}$ & $\mathrm{Ti}_{1}$ & $\mathrm{Te}_{1}$ \\
\hline$\Delta T_{2}$ & $V_{2}$ & $\mathrm{P}_{2}$ & $\mathrm{Ts}_{2}$ & $\mathrm{Ti}_{2}$ & $\mathrm{Te}_{2}$ \\
\hline$\Delta \mathrm{T}_{3}$ & $V_{3}$ & $P_{3}$ & $\mathrm{Ts}_{3}$ & $\mathrm{Ti}_{3}$ & $\mathrm{Te}_{3}$ \\
\hline$\Delta T_{4}$ & $V_{4}$ & $\mathrm{P}_{4}$ & $\mathrm{Ts}_{4}$ & $\mathrm{Ti}_{4}$ & $\mathrm{Te}_{4}$ \\
\hline$\Delta T_{5}$ & $V_{5}$ & $P_{5}$ & $\mathrm{Ts}_{5}$ & $\mathrm{Ti}_{5}$ & $\mathrm{Te}_{5}$ \\
\hline$\Delta T_{6}$ & $V_{6}$ & $P_{6}$ & $\mathrm{Ts}_{6}$ & $\mathrm{Ti}_{6}$ & $\mathrm{Te}_{6}$ \\
\hline$\Delta T_{7}$ & $V_{7}$ & $P_{7}$ & $\mathrm{Ts}_{7}$ & $\mathrm{Ti}_{7}$ & $\mathrm{Te}_{7}$ \\
\hline$\Delta T_{8}$ & $V_{8}$ & $P_{8}$ & $\mathrm{Ts}_{8}$ & $\mathrm{Ti}_{8}$ & $\mathrm{Te}_{8}$ \\
\hline$\Delta T_{9}$ & $V_{9}$ & $\mathrm{P}_{9}$ & $\mathrm{Ts}_{9}$ & $\mathrm{Ti}_{9}$ & $\mathrm{Te}_{9}$ \\
\hline$\Delta \mathrm{T}_{10}$ & $V_{10}$ & $P_{10}$ & $\mathrm{Ts}_{10}$ & $\mathrm{Ti}_{10}$ & $\mathrm{Te}_{10}$ \\
\hline$\vdots$ & & $\vdots$ & $\vdots$ & $\vdots$ & $\vdots$ \\
\hline$\Delta T_{n}$ & $V_{n}$ & $\mathrm{P}_{\mathrm{n}}$ & $\mathrm{Ts}_{\mathrm{n}}$ & $\mathrm{Ti}_{\mathrm{n}}$ & $T_{n}$ \\
\hline
\end{tabular}

Com o auxílio de softwares comerciais (Statistica e Excel), foi realizada uma regressão múltipla com o intuito de obter uma equação que apresente uma maior correlação comos dados coletados. O resultado foi uma equação da perda térmica em função das variáveis analisadas.

\section{RESULTADOS E DISCUSSÃO}

Em um primeiro momento, faz-se necessário identificar o problema atual para, posteriormente, analisar as melhorias provocadas pela construção do modelo de perda térmica proposto. O modelo de perda térmica utilizado anteriormente na thyssenkrupp CSA é uma taxa $\left({ }^{\circ} \mathrm{C} / \mathrm{min}\right)$ linear. Ou seja, considera-se a perda térmica como função apenas do tempo de espera para carregamento da panela. A figura 5 relata o problema decorrente da utilização do modelo de perda térmica atual. 


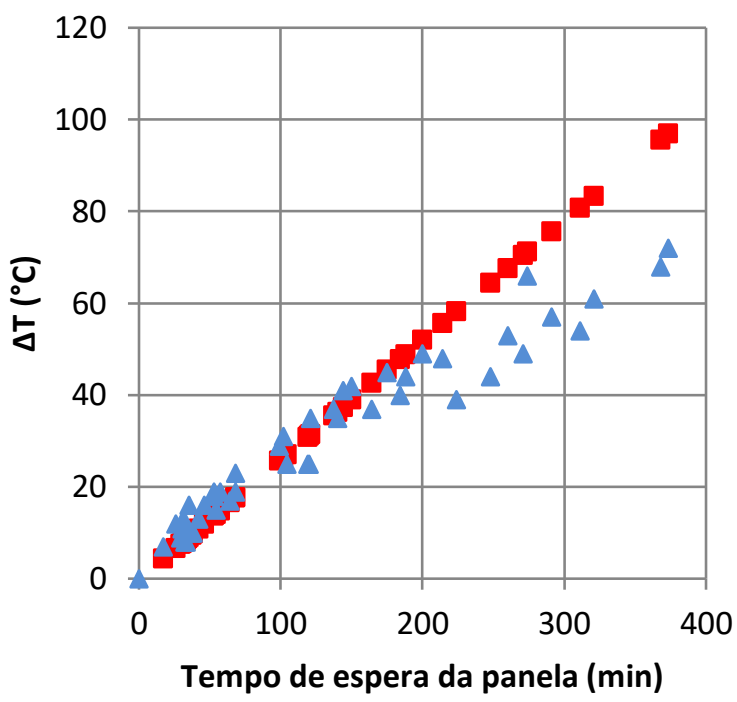

Valores previstos modelo atual

$\triangle$ Valores reais

Figura 5: Desvio entre os valores de perda térmica reais e previstos pelo modelo atual

A figura 5 demonstra que os valores de perda térmica calculados pelo modelo atual linear apresentam um erro considerável em relação à perda térmica real. Em detalhes, pode-se observar que para tempo de espera da panela até aproximadamente 200 minutos, a previsão de valores de perda térmica é satisfatória. No entanto, torna-se claro, a partir deste tempo de espera, que a função perda térmica perde sua linearidade e, consequentemente, o modelo de previsão da perda térmica utilizado perde a assertividade. Considerando estes erros do modelo atual, foi proposto um novo modelo de perda térmica.

A partir dos dados coletados, computados na tabela 1, realizou-se uma regressão múltipla no Microsoft Excel. A saída computacional é mostrada na tabela 2.

Tabela 2: Saída computacional regressão múltipla

\begin{tabular}{|l|r|}
\hline \multicolumn{1}{|c|}{ Estatística de regressão } & \\
\hline R múltiplo & 0,98163 \\
\hline R-Quadrado & 0,96361 \\
\hline R-quadrado ajustado & 0,95828 \\
\hline Erro padrão & 3,83834 \\
\hline Observações & 48,00000 \\
\hline & \\
\hline & Coeficientes \\
\hline Interseção & A \\
\hline Vida da panela & B \\
\hline Peso bruto da panela & C \\
\hline Tempo da panela sem gusa & D \\
\hline Temperatura inicial & E \\
\hline Tempo de espera da panela & F \\
\hline $\begin{array}{l}\text { Tempo de espera da } \\
\text { panela^2 }\end{array}$ & G \\
\hline
\end{tabular}


Nota-se, na tabela 2, a presença de uma variável denominada Tempo de espera da panela^2. Esta variável foi adicionada aos dados com o intuito de corrigir a não linearidade, em relação ao tempo de espera da panela, apresentada pelos valores de perda térmica reais. A partir dos coeficientes da saída computacional, pôde-se construir a equação de perda térmica do modelo proposto (Equação 1).

$$
\begin{array}{r}
\Delta T=A+B * V i d a+C * \text { Peso }+D * \text { Tempo sem gusa }+E * \text { Temperatura inicial }+ \\
F * \text { Tempo de espera }+G * \text { Tempo de espera } 2(1)
\end{array}
$$

Destaca-se o valor do $R^{2}=0,958$. Esta medida de ajustamento do modelo estatístico linear é um meio de validar a regressão. Portanto, como o valor para $R^{2}$ foi bastante próximo da unidade, conclui-se que a regressão realizada foi satisfatória.

A partir da equação, foi plotado o gráfico apresentado na figura 6. A figura 6 faz uma comparação entre os valores de perda térmica reais, valores previstos pelo modelo atual e valores previstos pelo modelo proposto.

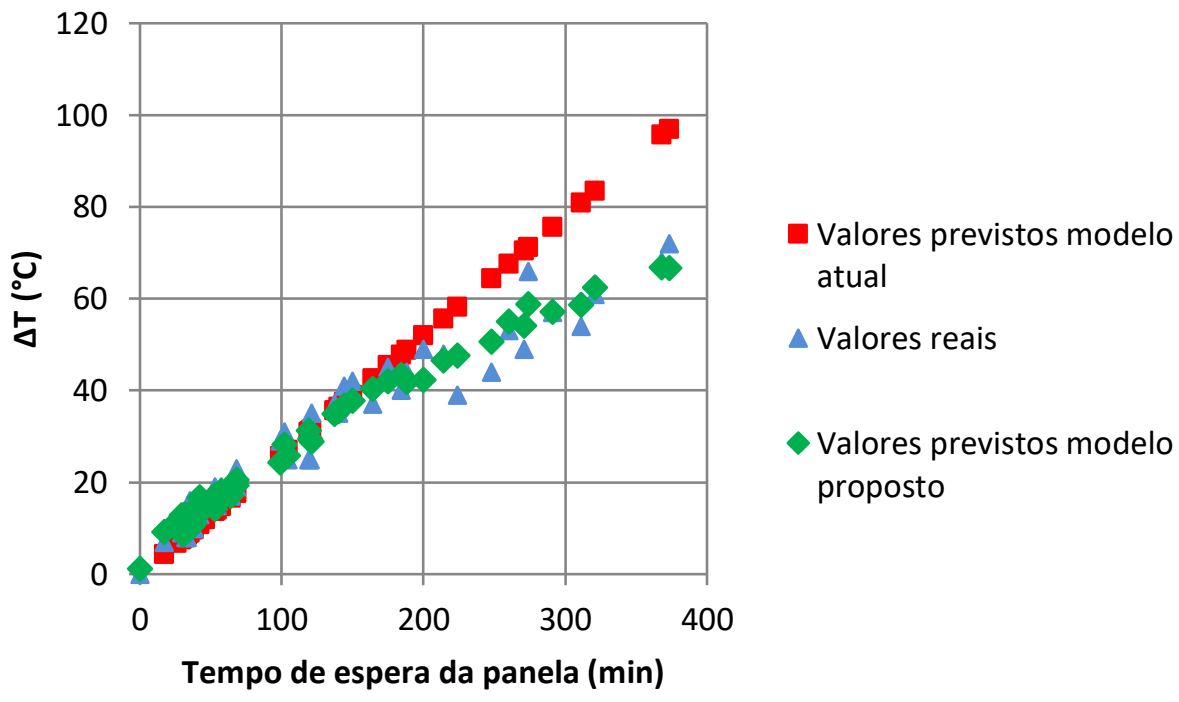

Figura 6: Comparação valores de perda térmica

Através de análise gráfica, os resultados sugerem uma maior aproximação entre os valores de perda térmica reais e os valores previstos pelo modelo proposto. Ou seja, há uma maior assertividade do modelo proposto em relação ao modelo atual de perda térmica na panela de gusa utilizada na thyssenkrupp CSA. O gráfico da figura 7 foi plotado, adicionalmente, para ilustrar esta maior assertividade do modelo proposto. Observa-se, neste gráfico, que a linha de tendência dos valores previstos pelo modelo proposto sugere uma maior aproximação entre estes valores e os valores de perda térmica reais. Isto pode ser observado pela análise dos coeficientes de correlação de ambas as retas. 


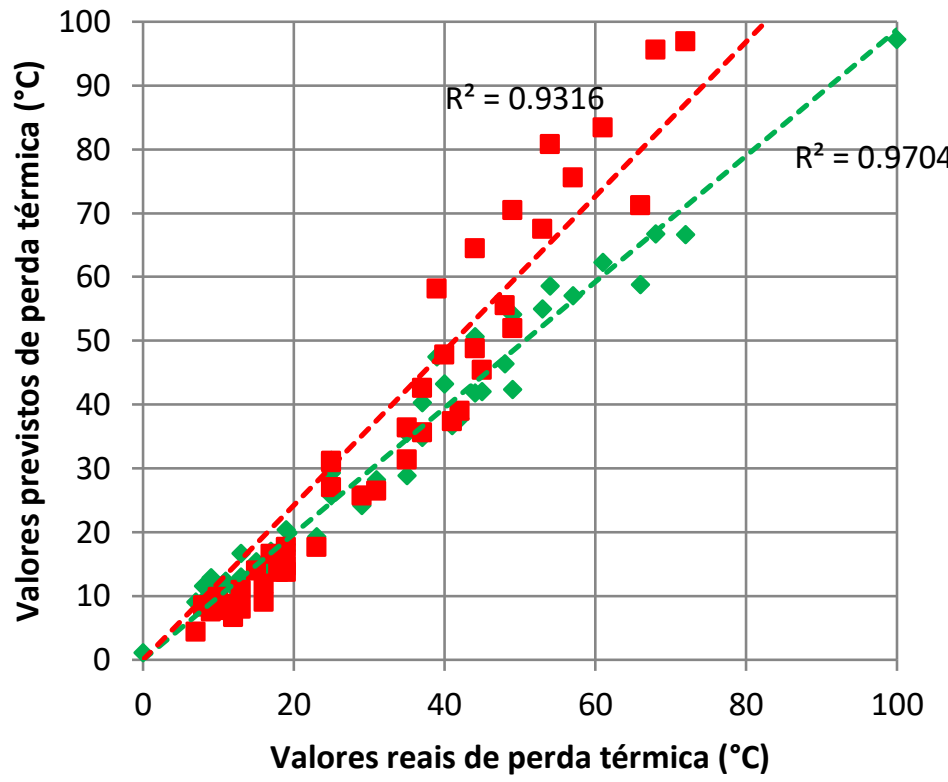

- Valores previstos modelo proposto

- Valores previstos modelo atual

Figura 7: Comparação da assertividade dos modelos proposto e atual

Com o objetivo de validar o modelo proposto, foi realizada uma análise de sensibilidade das variáveis consideradas no software Statistica®. A análise de sensibilidade está relacionada ao nível de influência de cada variável na perda térmica. A figura 8 mostra esta análise.

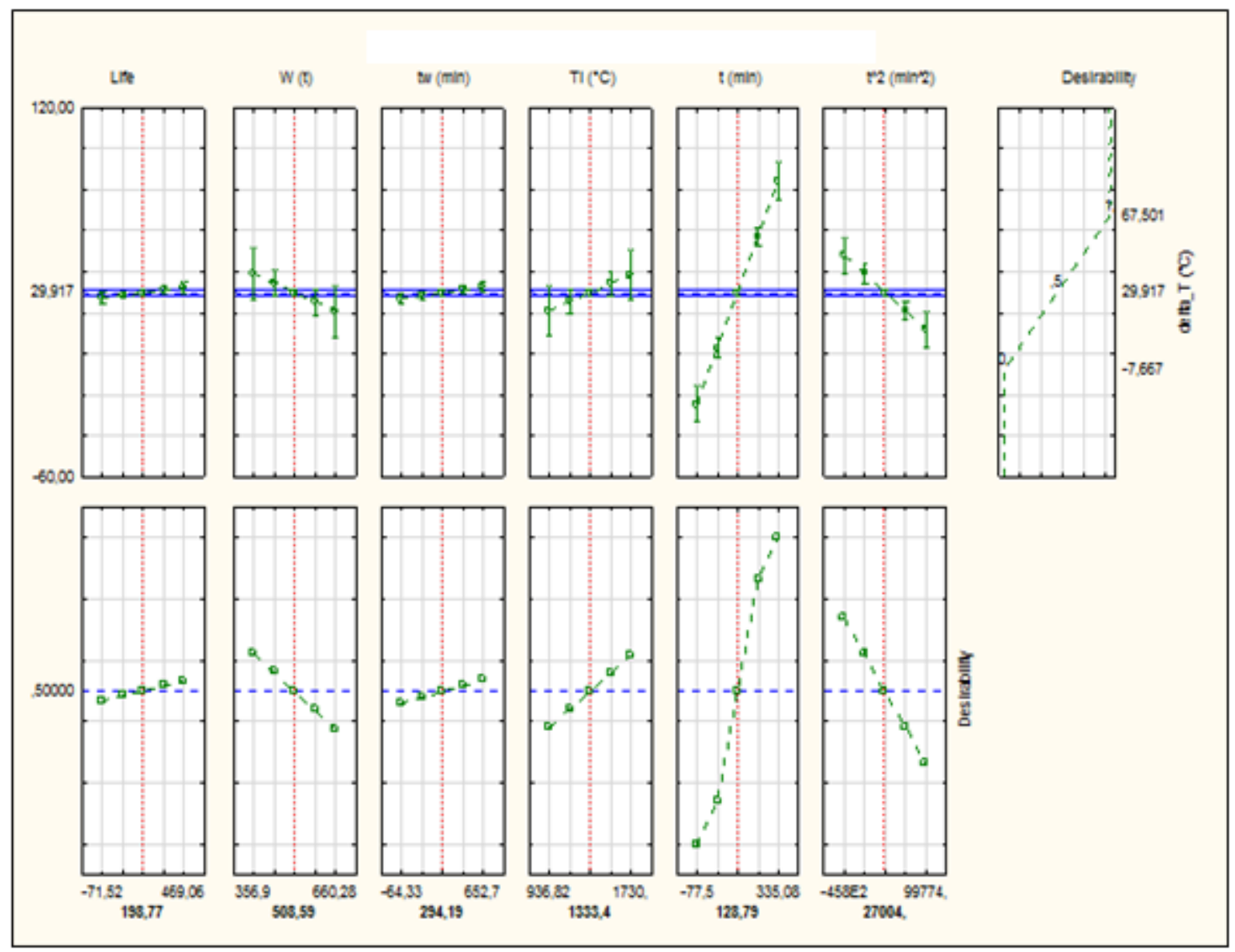

Figura 8: Análise de sensibilidade das variáveis 
A partir da Figura 8, tem-se o tempo de espera da panela de ferro-gusa (t) como a variável mais influente na perda térmica. Ao mesmo tempo, no entanto, vale ressaltar a influência das outras variáveis consideradas. A vida da panela ("Life") e o tempo da panela sem gusa (tw) têm menor influência enquanto o peso bruto da panela (W) e a temperatura inicial do ferro-gusa pós dessulfuração (Ti) apresentam uma influência intermediária na perda térmica.

Outro ponto que vale destaque está de acordo com a análise dos resíduos dos modelos. O resíduo consiste na diferença entre o valor previsto pelo modelo e o valor de perda térmica real. Sabe-se que a normalidade dos resíduos é um meio de validação da regressão. A figura 9 indica que os resíduos do modelo proposto seguem a normalidade e, portanto, conclui-se que a regressão realizada é válida.

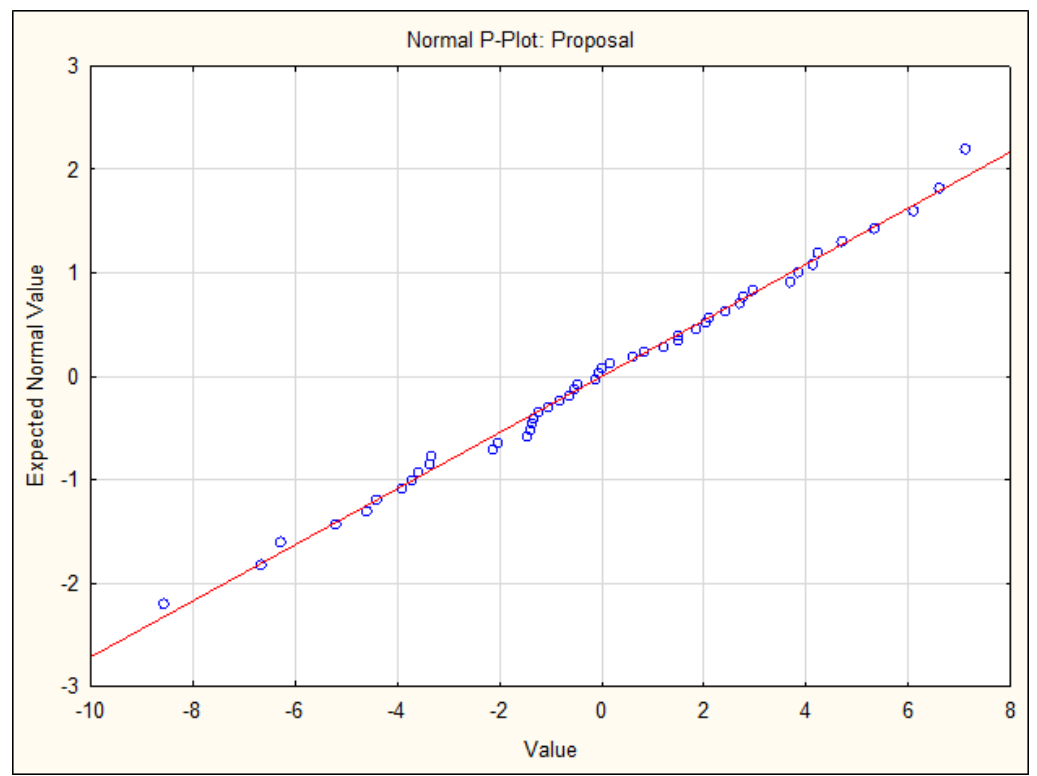

Figura 9: Normalidade dos resíduos da regressão múltipla

Além disso, a figura 10 faz uma comparação entre os resíduos dos modelos atual e proposto de perda térmica. Pode-se observar que a média dos resíduos do modelo proposto é centrada no valor zero e que o intervalo de não outliers (definido como um ponto que está muito distante das demais observações em uma série estatística) é menos extenso que o intervalo de não outliers dos resíduos do modelo atual. Este Box Plot (gráfico utilizado para avaliar a distribuição empírica dos dados) realça a menor variabilidade alcançada pelo modelo de perda térmica proposta em relação ao modelo atual. 


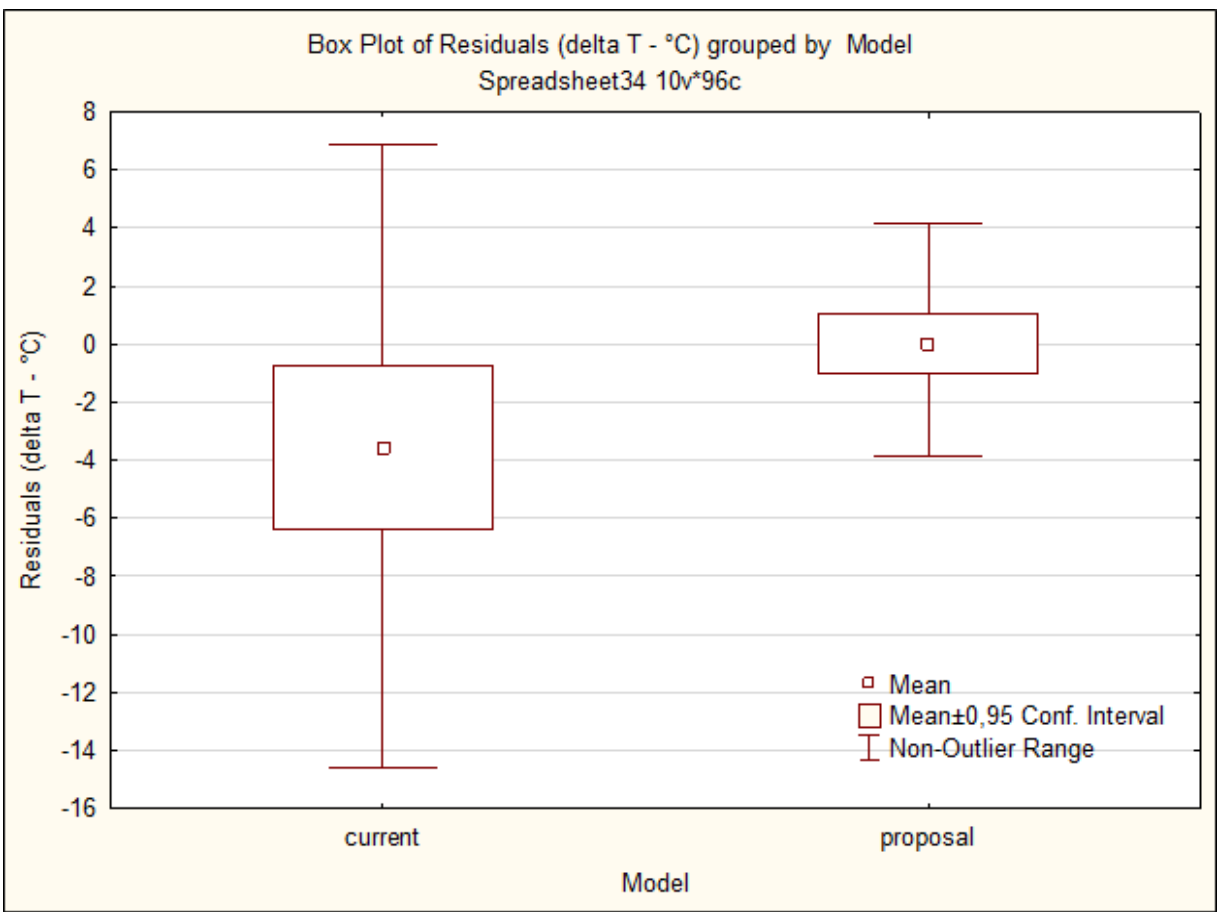

Figura 10: Box Plot dos resíduos dos modelos proposto e atual

Vale ressaltar que o modelo proposto ainda não está em uso na thyssenkrupp CSA e, portanto, ainda não existem resultados práticos.

\section{CONCLUSÃO}

Destacam-se as conclusões:

- Importância do acerto da temperatura de carregamento do ferro-gusa. Erros na temperatura de carregamento acarretam em balanços térmicos equivocados, o que pode interferir negativamente na corrida.

- Relevância das variáveis vida da panela, peso bruto da panela, tempo de espera da panela e temperatura inicial do ferro gusa. Estas variáveis influenciam diretamente no processo de perda térmica na panela de ferrogusa.

- Modelo de perda térmica proposto apresenta maior assertividade do que o modelo de perda térmica utilizado atualmente na thyssenkrupp CSA.

\section{Agradecimentos}

O presente trabalho teve apoio financeiro e prático da thyssenkrupp CSA. Faz-se necessário agradecer aos idealizadores deste projeto, especialmente aos engenheiros metalurgistas da aciaria da CSA.

\section{REFERÊNCIAS}

1. FERREIRA, N. F., et al. INFLUÊNCIA DO DESGASTE DE PANELAS SOBRE AS PERDAS TÉRMICAS DO AÇO LÍQUIDO. In: Anais do 35 Seminário de Fusão, Refino e Solidificação dos Metais, Salvador, 2004. 
2. LOPES, H., L., P. MODELO PARA PREVISÃO DA CONDIÇÃO TÉRMICA DE PANELAS DE ACIARIA.Dissertação de Mestrado apresentada ao Curso de Pós-Graduação em Engenharia Metalúrgica e de Minas da Universidade Federal de Minas Gerais. Dezembro.

3. MUNDIM, M. J., et al. ANÁLISE TÉRMICA DA PANELA DE AÇO. In: Anais do 40ํㅡㄹ Congresso Anual da ABM, Rio de Janeiro, 1985.

4. TETRAULT, C., et al. CFD Analysis of Thermal Behavior of Refractory in Steel Ladle Cycling. AISTech 2004 Proceedings, Volume I, 2004 